\title{
RFID-Enabled Real-Time Manufacturing for Automotive Part and Accessory Suppliers
}

\author{
George Q. Huang, T. Qu, YingFeng Zhang \\ Dept of Industrial and Manufacturing Systems Engineering, \\ The University of Hong Kong, \\ Hong Kong, China (GQHuang@hku.hk)
}

\author{
HD Yang \\ Faculty of Automation, \\ South China University of Technology, \\ Guangzhou, China
}

\begin{abstract}
Automotive part and accessory manufacturers (APAMs) at the lower tiers of the automotive vertical have been following leading vehicle assemblers in adopting RFID (Radio Frequency Identification) and ubiquitous computing technologies, aiming to alleviate their advanced manufacturing systems. RFID-enabled real-time traceability and visibility facilitate the implementation of advanced strategies such as JustIn-Time (JIT) lean / responsive manufacturing and mass customization (MC). Being typically small and medium sized, however, APAMs are faced up with business and technical challenges which are summarized by the so-called "three high problems". They are high cost, high risk and high level of requirement for technical skills. Based on a series of industrial field studies, this paper establishes an innovative service-oriented business model for overcoming the "three high Problems" based on the concept of Product Service Systems (PSS) and RFID gateway technology.
\end{abstract}

Keywords- automotive part and accessory manufacturers, automotive alliances, RFID / auto id, real-time manufacturing, manufacturing execution system

\section{INTRODUCTION}

RFID (Radio Frequency Identification) or Auto-ID (Automatic Identification) has received substantial attention in supply chain logistics (SCL) since the Wal-Mart mandate (Williams 2004). This is particularly true as a result of the initial aggressive promotion and developments, mainly through the Auto-ID Labs (http://www.autoidlabs.com) and recent worldwide efforts made by both industrialists and academics. The rapid drop in the costs of higher frequency RFID tags and readers has made automated data collection - without the limit of "line of sight" - economically viable and affordable. It is now possible for RFID technology to eliminate a large number of manual business process transactions and associated manual data collection/entry in supply chain management (SCM).

Product manufacturers, situated at the upstream of the global supply chain, have critical roles to play in making RFID a success in SCL. There has been increasing evidence that manufacturing enterprises are picking up RFID-based solutions quickly, not only to meet the mandates but also to gain benefits in productivity and quality improvements (RFID Journal 2005; Huang et al 2009). This is because RFID sensors are an effective means for collecting and processing field data from manufacturing machines, thus enabling responsive production planning and control (Osman and Furness 2000). Huang et al (2009) has proposed the concept of Wireless Manufacturing
(WM) as an umbrella term for manufacturing solutions enabled by wireless devices such as RFID and other types of wireless devices. The long-term objective of WM is to develop a nextgeneration advanced manufacturing technology (AMT) with core technological building blocks including wireless devices (e.g. RFID / Auto-ID) and wireless information and communication networks (e.g. Wi-Fi, Bluetooth, and ZigBee) for the collection and synchronization of real-time field data from manufacturing workshops. WM replaces paper-based manual shopfloor tracing and tracking systems with wireless smart devices. This will ultimately improve and optimize the shop-floor operational efficiency and effectiveness. WM thus also closes the loop of production planning and control by providing real-time feedback of such sensory data for adaptive decision making.

The research reported in this paper is concerned with RFID-enabled manufacturing applications in automotive manufacturing. The focus is placed upon developing and deploying such solutions within lower tier automotive part suppliers down the automotive vertical. These suppliers are usually much smaller in terms of their business size / scope and financial powers as compared with vehicle assemblers and top tier component manufacturers.

The paper is organized as follows. First, a comprehensive literature review is conducted about RFID automotive manufacturing applications in Section 2. Second, field case studies within collaborating companies are reported in Section 3. Through the literature review and field studies, current situations and expectations are examined, and business and technical challenges are identified and analysed. In addition, methods for tackling the challenges are also observed from the field studies within collaborating companies.

\section{RFID-BASED APPLICATIONS IN AUTOMOTIVE MANUFACTURING}

RFID (Radio Frequency Identification) technology is not new to automotive industry (http://www.abiresearch.com). Early applications have been dominated by RFID-enabled vehicle entry and security. For example, up to $40 \%$ of new cars manufactured in North Americas are equipped with RFID immobilizers. In recent years, the interests in RFID have dug deeper along the automotive vertical from top-level automotive assembly lines to part and accessory suppliers at different levels of the automotive supply chains. Compelling opportunities and potential benefits for RFID usage in the automotive manufacturing processes have been widely 
highlighted by authoritative market intelligence reports (VDC; ABI Research). It is indeed encouraging to note recent RFID initiatives made by major automotive manufacturers.

Leading automotive manufacturers have been at the forefront of adopting RFID (Radio Frequency Identification) and ubiquitous computing technologies to alleviate their advanced manufacturing systems. For example, major automotive manufacturers (Strassner and Fleisch 2003; Gould 2000; Intermec Technologies 2001; RFID Journal 2004; Mintchell 2002) such as Volvo Trucks (Johansson 2004), Volkswagens (Identecsolutions 2004), Nissan (GlobalManufacture 2004), and Ford (Johnson 2002) have experimented with the ideas. Some recent attempts are summarized below.

Firstly, in early 2009, BMW initiated to employ an RFIDbased Tool Assistant System (TAS), provided by Ubisense, at its assembly plant in Regensburg, Germany. TAS helps to match the cars being assembled with the correct tools needed to perform the job, thereby automating a system to provide each vehicle with custom assembly, based on the car's vehicle identification number (VIN) (BMW 2009).

Secondly, Volkswagen, Europe's leading vehicle manufacturer, deployed the new system in 2009 following a one-year pilot project in which the automaker and IBM tested RFID technology with suppliers. Using this system, shipping containers carrying auto parts destined for Volkswagen will be increasingly fitted with RFID tags (Volkswagen 2009).

The third case that deserves a mention is that Aston Martin, a high-performance car manufacturer, used Ubisense RFID tags in 2009 to track the locations of vehicles as they move through the final steps in the company's manufacturing process to ensure they are produced according to the demands of the automaker's customers (Aston Martin 2009).

Fourthly, Jaguar and its logistics partner, Unipart Logistics, use sensors and ISO-standard active RFID tags on shipping containers as part of the Jaguar Tradelane Project to provide real-time location information and security alerts for shipping containers packed with replacement parts. Savi's systems at the Port of New York/New Jersey and the Port of Oakland in California will track inbound Jaguar shipments (Jaguar 2009).

Finally, Toyota has used RFID to realize asset management. They installed WhereNet Active RFID tags to vehicles and cargo containers. Through monitoring the locations of tags, they could ensure the right cargos has been loaded onto the right vehicles and delivered to customers according to the right schedule. WhereNet has large volume and price, about $\$ 40-80$ per piece, and could be used only to large vehicles and containers (Toyota 2009).

\section{INDUSTRIAL FIELD STUDIES}

The literature review above has revealed a gap between the opportunity and challenge for small and medium automotive part and accessory manufacturers (APAMs). On the one hand, these APAMs enjoy the opportunity to gain matching benefits of improved productivity, responsiveness and quality by adopting and integrating corresponding RFID-enabled advanced manufacturing technologies (AMT) with those of top-level vehicle assembly manufacturers. RFID-enabled realtime traceability and visibility across their assembly lines help them better realizing their advanced strategies such as Just-InTime (JIT) lean / responsive manufacturing and mass customization (MC). On the other hand, being typically small and medium sized, APAMs face financial and technological challenges to initiate and implement advanced RFID-enabled manufacturing solutions.

\section{A. Overview of Industrial Field Studies}

In order to reveal why such RFID technologies have not yet been widely used in practice across the automotive manufacturing sector, especially within lower tier part suppliers, this research conducts a number of industrial field studies within collaborating companies with the support a number of joint R\&D projects sponsored by regional government bodies, universities and private companies located in Greater Pearl River Delta (GPRD) of southern China. Many of the companies have business focuses on automotive part manufacturers (APAMs) and logistics service providers. Special attention has been paid to two sets of automotive part manufacturers who have formed automotive alliances located in two corresponding geographical areas. The first alliance (noted as metal alliance of APAMs subsequently) includes APAMs located in one city and most of them have been involved in manufacturing automotive metal parts and components mainly used for assembling auto engines. The other set (noted as electronic alliance APAMs subsequently) consists of APAMs located within an industrial park / estate and they are mostly involved in manufacturing automotive electronic parts and components. The metal alliance is let and hosted by a leading member while the electronic alliance has a loose structure as members are managed under the same industrial park.

The scope of field studies involves the complete material flow lines. Typically, the team started backwards with key business processes and operations from warehouses and dispatching areas of finished products through final product assembly lines, component subassembly lines, WIP (Work In Progress) inventories, and raw material inventories. The team also investigates their existing EISs (Enterprise Information Systems) such as ERP, WMS, PDM or any IT supports in operation. As most companies have thought about RFID technologies before the studies, the team also exchanged ideas on how far the companies have moved towards RFID adoption, what motivate and/or hinder their further efforts, and what supports they expect the RFID technologies to provide. The team also gave presentations during the discussions to share the experiences that have been gained from different projects and companies.

Field study companies can be divided into three levels. Level 1 companies are those who are considering introducing RFID. Let us call them RFID "shoppers". Level 2 companies are those who have already started their pilot RFID projects, gained certain insights and benefits, and are considering expanding their efforts. Let us call them RFID "starters". Majority of our field study companies are starters. Level 3 companies are those that have introduced RFID in their key 
processes and operations for some time, and are at the stage of comprehensively utilizing RFID-enabled real-time visibility and traceability. Let us call them RFID "pioneers". Very few in our field studies are pioneer companies.

Findings from the field studies can be grouped under three categories: (1) problems that can be potentially solved by RFID-enabled manufacturing solutions, (2) reasons why automotive part manufacturers are reluctant towards adopting RFID-enabled manufacturing solutions, and (3) hints how their business and technical concerns should be adequately addressed.

\section{B. Motivations for Considering RFID-Enabled Shopfloor Solutions}

There are several typical motivations for field study companies to consider RFID-enabled shopfloor solutions. Some of them are summarized as follows:

- One push is from the top tier vehicle assemblers who have reportedly experimenting with adopting RFID solutions in their Just-In-Time (JIT) assembly lines. Lower tier suppliers would like to get ready before their upper tier customers mandate them to do so. Pulling effects come from business and operational problems that these companies are faced.

- From the business perspective, profit margin of APAMs is therefore largely squeezed. It is known that only $5-10 \%$ of production time and $20 \%$ of production cost are spent on the physical manufacturing process, while $90-95 \%$ and $80 \%$ of those are on the material storage, transportation and management (Manufacturing Business Technology, 2009). Therefore, APAMs have to further optimize the logistics processes to cut costs and finally survives.

- An increasing number of APAMs have been implementing IT-based decision support systems (e.g. ERP, WMS) to elevate their capability of dealing with business information, a bottleneck problem is the data collection of manufacturing logistics. Enterprise decision systems may suffer from the so-called "Garbage in, Garbage out" syndrome. Plans and schedules do not reflect the shopfloor reality and therefore cannot be duly executed.

- From a supply chain perspective, lower-tier part manufacturers are normally smaller in size and farther away from end markets. Therefore, they suffer deeper from the so-called bullwhip effect. They are generally willing to collaborate with their upper-tier manufacturers to share information as far as possible to reduce the bullwhip effects.

- APAMs located in the PRD region are at the upstream of the automotive supply chain, typically at 2 nd and 3rd tiers or even further. They are extremely sensitive to frequent changes in the top-level automotive assembly plants and the global market \& supply base as well as national and local policies.
- The inability for APAMs to achieve responsive manufacturing as demanded by Just-In-Time (JIT) manufacturing strategy of the top-level automotive manufacturers is largely caused by delays and errors in shop floor production and logistics data which are often inconsistent, incomplete, incompatible, inaccurate and untimely.

- The information infrastructure to support RFIDenabled APAMs is inadequate. Companies are still figuring out basic questions such as where RFID readers and tags should be deployed and which frequency is best for the application. There is no systematic scheme for integrating the RFID devices seamlessly to automotive manufacturing processes.

- APAMs, even some existing RFID users in the manufacturing sector, may have basic understanding on how RFID generally works in their manufacturing processes. Few of them have clear ideas on how realtime information can be used to improve their internal operations and decisions, nor inter-enterprise collaboration and sharing. Only some advanced RFID users have started the next wave of real-time data mining, for example, for determining standard operations times to be used for production scheduling.

\section{C. "Three high problems"}

The field studies have found out reasons why automotive part manufacturers are reluctant towards adopting RFIDenabled manufacturing solutions. Reasons can be summarized under the following three categories or the so-called "three high problems." They are (1) High cost, (2) High risk, and (3) High level of specialist technical skills.

\section{1) High Cost}

The first and foremost concern about the RFID adoption is that the capital threshold of RFID solutions has been widely perceived as high. Concerns about high costs of adopting RFID solution change with the experiences and stage of the RFID adoption project, for example for beginner, intermediate and advanced companies as follows:

- $\quad$ Starter companies (at the beginning stage of adopting RFID solutions) are more concerned about direct costs spent on acquiring RFID devices and tags. The reason is that they have little experience of an optimal deployment scheme minimizing the number (and thus the cost) of RFID readers. Early adopters have initially received supports by participating in governmentsponsored R\&D projects.

- Intermediate companies (those have accumulated certain experience and insights of using RFID solutions) are mainly concerned about several cost factors other than reader costs. For example, they are aware of the indirect cost involved in streamlining their business processes and operations after adopting RFID solutions. Another cost driver is the integration and interfacing with their existing decision support systems (DSSs) such as ERP, WMS and MES. As integration requires high level of technical skills, the cost could be 
high. Finally, they are at the stage of dealing with the cost driver of providing technical services and supports to RFID devices and solutions.

- In contrast, advanced RFID pioneers are investing in extending and integrating the RFID-enabled solutions into and with other enterprise decision support and information systems in the value chain. They recognize that the maximum benefits are achieved when their business partners (e.g. suppliers and outsourcing contractors) are also using RFID solutions.

\section{2) High risk}

RFID technologies have developed rapidly. RFID consultants have been telling manufacturers that "new RFID devices and solutions are introduced every year and RFID devices acquired a year ago have now become obsolete and outdated." This has created enormous concerns even risktaking manufacturers. Our field studies have revealed some stories:

- Simple applications developed for one handheld device under an old operating system do not work properly under a new version of the operating system.

- Cost and capabilities of RFID devices acquired in between 12-24 months are different. Manufacturers tend to wait for a little more while for the next generation of RFID devices.

- The upgrading of devices and software systems may not be synchronized and cannot accommodate each other. What worry SMEs even more is that RFIDrelated standards evolve so rapidly for them to comprehend and follow.

- Although the reliability and readability of RFID devices have dramatically been improved in the last 5 years, there are still significant limitations for automotive manufacturing applications where 6-sigma strategy has been widely adopted.

3) High level of specialist technical skills

RFID solutions require very high level of specialist knowledge and practical skills in Information Technology (IT), RFID readers and business processes and operations. Technical concerns are multi-folded:

- Engineers / technicians from IT and operations departments have little knowledge and skills with RFID technology, even after attending seminars and short-course training. It takes them significant efforts in setting up the first demonstration, usually led by the RFID technology provider. Therefore, the companies believe that it is more expensive to invest in acquiring RFID know-how skills than acquiring RFID devices.

- The technical contents of the RFID system are complicated and difficult for technicians in individual companies to deal with cost-effectively. Taking RFID readers as an example, they could be categorized into low-frequency, high-frequency, ultra-high-frequency and microwave types by the utilized frequency bands, while into fixed, portable and handheld by their usages.
Different manufacturing environments may require different types of readers according to their specific requirements and constraints (as have shown in the scenarios). The RFID tags attached to the associated manufacturing resources will thus be different as well.

- The selection of appropriate RFID devices dominates the early stage before a project proceeds to a stage of full-scale application.

- The configuration and operation of RFID devices and components is complicated. So far, however, due to the lack of uniform standards and compatible software, RFID and other Auto-ID devices cannot be used in a "Plug-and-Play" mode. Different readers normally have different drivers applied to different operating systems, and thus the development methods are totally different. At the same time, the failure diagnosis and maintenance for RFID devices will be much timeconsuming, especially when large amount of devices are used.

\section{Hints from Industrial Field Studies}

The field studies not only identified the key challenges as discussed above, but also obtained several crucial hints how such business and technical challenges could be adequately addressed.

1) Two Approaches to Solving "Three High Problems"

The first crucial finding is that the above so-called "three high problems" can be solved by two approaches. One is "reduction" and the other is "sharing". Significant progresses have been made in the "reduction" approach. For example, RFID device and tag manufacturers have been able to substantially reduce the cost of RFID devices and tags. The risk and level of technical skills have also been significantly reduced through new standards at the hardware and software levels.

However, the extent of the "reduction" approach is limited and manufacturers, especially small and medium sized enterprises (SME), may still be unable to afford the RFID costs. Then the "sharing" approach becomes more relevant. There are two types of sharing. One is for RFID solution developers and providers to share the cost, risk and technical skills with the end-users. This has become increasingly a common business model called Product Service System (PSS). The other type of sharing is among end-users that belong to the same interest group (for example a business alliance) and use similar RFID solutions.

\section{2) Sharing AUTOPS SaaS is Feasible among Automotive} Suppliers

The second crucial finding is that many automotive part and accessory manufacturers (APAMs) often work together in some forms of alliances (APAMA). Such alliances take the two typical forms: vertical partnership and lateral association. A vertical partnership exists between a key automotive component manufacturer and its strategic suppliers of key parts in the entire vehicle supply chain. A strategic partnership usually exists between a vehicle manufacturer and its 1st-tier suppliers of key components such as engines, body works, 
frameworks, etc. used for the final assembly. In turn, a partnership exists between a 1st-tier supplier and lower-tier part suppliers. Lower-tier part suppliers may establish partnerships with more than one upper-tier component manufacturers.

For example, our field studies have involved a number of manufacturers of automotive metal parts (such as valves, engine bodies, piston rods, etc.) located in the same city for auto engine manufacturers. These part manufacturers take orders from engine makers all over the world. Recently, they formed an alliance which is more or less the vertical partnership scenario. In a vertical alliance, members are more business partners than competitors. They share incentives of mutual collaboration (e.g. sharing information) in improving productivity, quality and reducing costs and lead times. Because they are business partners, there are more instruments to stimulate mutual collaboration through sourcing and pricing policies of the parts and related services.

A lateral association is an alliance of automotive part and accessory manufacturers located at the same tier in the automotive vertical supply chain. Members of the alliance are related to each other through some common factors such as operating within a geographical proximity or sharing similar manufacturing technologies / capabilities or producing similar automotive parts and accessories (e.g. automotive electronic components). Therefore, members of the alliance are potentially competitors to each other as they may compete for orders of the same parts from the same customers. However, they are equally motivated to locate and collaborate close to each other for several reasons.

A number of field studies have been carried out within an industrial park located in the PRD region. This industrial park is dominated by electronics product manufacturing companies, a noticeable proportion of which are manufacturers of automotive electronics parts and accessories. Many of them are SMEs and they have limited manufacturing resources and production capacities. A lead APAM will take advantage of the collective capabilities and capacities in order to secure large customer orders which cannot be won by any individual APAMs in the alliance alone. It is common for the members of this coalition to share out the successful large customer orders. Similarly, APAMs are able to share raw materials, semifinished products, even inventory spaces. As a result, transportation and inventory costs can be substantially reduced within the alliance. In addition, by being located at a geographical proximity, APAMs are able to dynamically and quickly identify human skills without requiring workers to relocate themselves.

Individual APAMs of the above two forms of alliances are highly motivated to participate in corresponding alliances in order to gain following benefits: (1) Share market information, (2) Share technology, (3) Share capability and capacity, (4) Share logistics and other types of services, and (5) Share investments.

Geographically, APAMs in alliances may be located closed to each within a so-called automotive manufacturing base or industrial park / estate, or enjoy virtual relationships managed by the most influential (lead) enterprise in the alliance.
Automotive alliances are regulated and coordinated by the management offices of the corresponding industrial estates / parks or lead members of the alliances. This organizational structure of automotive alliances provides a foundation for institutionalizing service-oriented platforms which are provided by the lead members and shared by all members of the alliances. Through sharing, individual alliance members are able to reduce the cost, risk and technical level of adopting RFID solutions.

\section{3) Sharing AUTOPS SaaS is Feasible among Automotive} Suppliers

The next crucial hint is more technically oriented. During the field studies, the success story of Hong Kong Octopus (a variation of RFID technology) services was explained to the subject company. The objective of telling this story is to test if a similar technical and business solution framework could be considered within automotive alliances.

As early as in 1994, Hong Kong's five major public transport operators - the MTR, KCRC, KMB, Citybus and the Hongkong and Yaumatei Ferry (HYF) established a joint venture known as Creative Star Limited (renamed Octopus Cards Limited in 2002) to oversee the development and implementation of contactless smart card technology. In 1997, the Octopus fare collection system was officially launched. The innovative system eliminated the need for commuters to find exact change. It also allowed them to travel across multiple public transport modes using a single card. Since then, Octopus has been expanded into major retailer shops, canteens and restaurants. Over 10 million Octopus cards are in circulation for a 7 million population in Hong Kong. The Octopus technology has been successfully working for an alliance of major transport companies and retailers in Hong Kong. Octopus has become a pioneer of the world's leading and most widely used contactless smart card payment systems. Octopus is now an integral part of everyday life in Hong Kong.

The feedback from field studies is that the Octopus business model deserves serious consideration in manufacturing industries in general and automotive part manufacturing alliances in particular. Just like the Octopus consortium in Hong Kong, each automotive alliance sets up an Octopus-like service provider that operates the AUTOPS services. The rest of this paper focuses on discussing how the Octopus platform can be transplanted as the AUTOPS platform that is shared among members of automotive part manufacturing alliances.

However, such transplant has to deal with fundamental differences between the two application scenarios. For example, Octopus cards are essentially a form of electronic cash. Therefore, Octopus services can be easily charged according to the monetary transaction amounts. In contrast, RFID tags in the AUTOPS platform are not electronic cashes. The charging plans, i.e. members' contributions to the operation and maintenance of AUTOPS services, are complicated. Furthermore, Octopus cards are used as tickets for customers to enter or exit - the operations are straightforward. In contrast, RFID tags in AUTOPS are associated with multiple types of objects including human operators, materials and 
assets in manufacturing shopfloors. Associated information are complex in terms of their formats and contents.

\section{CONCLUDING DISCUSSION}

This paper has focused on discussing key issues related to the development and deployment of RFID applications in automotive part manufacturing industry. It has been widely acknowledged that RFID-enabled real-time visibility and traceability are able to enhance the implementation of advanced manufacturing strategies and technologies (e.g. lean manufacturing, mass customization, responsive manufacturing, etc) that have been playing essential roles in the automotive sector. However, significant challenges, both technical and business, exist especially within small and medium sized manufacturers at the lower tiers of the automotive vertical supply chains. A series of field studies have been carried out among existing and potential industrial collaborators to examine what exactly are the hurdles that have been hindering the RFID implementation and how best they can be addressed cost-effectively. Key challenges can be summarized as "three high problems", i.e. concerns about high initial investments and medium-term commitments, high level of technical risks of the rapidly developing technologies, and high level of specialist skills in acquiring and maintaining RFID solutions. The field case studies have observed some useful insights that provide essential hints of overcoming these "three high problems". Lower-tier automotive manufacturers tend to form alliances within which members collaborate with, though often also compete against, each other. With the concept of Product Service System, members of an automotive part manufacturing alliance are able to share RFID-enabled services, without separate investments in RFID skills and all components of RFID-enabled solutions.

\section{ACKNOWLEDGEMENTS}

We are most grateful to various companies who provide technical and financial supports to this research. Authors would like to acknowledge financial supports from HKSAR ITF (GHP/042/07LP) and HKSAR RGC GRF (HKU 712508E).

\section{REFERENCES}

[1] ABI Research, http://www.abiresearch.com/

[2] Aston Martin (2009) http://www.rfidjournal.com/article/view/5296/

[3] Gould, L.S. (2000), "What you need to know about RFID", Automotive Manufacturing \& Production, Vol. 112 No.2, pp.46-9.

[4] Hong Kong Octopus, http://www.octopus.com.hk/octopus-forbusinesses/octopus-technology/en/index.html

[5] Huang, GQ., Wright, PK., Newman, ST., 2007, Wireless Manufacturing: a literature review, recent developments and case studies, Internationa Journal of Computer Integrated Manufacturing, Volume 22, Issue 7 July 2009 , pages $1-16$.

[6] Identecsolutions (2004) IDENTEC SOLUTIONS RFID System Tracks Volkswagens for Europe's Largest Auto Maker, Available online at http://www.identecsolutions.com/VW.asp (Accessed on 20 March 2010).

[7] Intermec Technologies, RFID Applications for the Automotive Industry, November 2001

[8] Jaguar (2009) http://www.rfidjournal.com/article/view/2676

[9] Johansson, M., Identification of the main factors influencing an RFID implementation in the automotive and pharmaceutical industries, Linkopings University, 2005.

[10] Johnson D., 2002, "RFID tags improve tracking, quality on Ford line in Mexico," Control Engineering, 49 (11), pp. 16-16.

[11] Mintchell, G. (2002). It's automatic: automation shifts transmission assembly into high gear, Control Engineering, 49(6), 12.

[12] Osman KA, Furness, A., "Potential for two-dimensional codes in automated manufacturing," Assembly Automation, 2000, vol. 20, no. 1, pp. 52-57(6). Publisher: Emerald Group Publishing Limited.

[13] RFID Journal, (2004), RFID Supply Chain Solution Provider Introduces a System That Tracks the Quantity of Automotive Parts on the Assembly Line and Reorders When Stocks Are Low, RFID Journal. Available online at http://www.rfidjournal.com/article/articleview/807/1/26/. (Accessed on 20 March 2010).

[14] Strassner, M. and Fleisch, E. (2003) The Promise of Auto-ID in the Automotive Industry, White paper of AUTO-ID Center in MIT, Available online at: http://www.autoidcenter.org/ research.asp. (Accessed on 20 March 2010).

[15] Toyota (2009) http://www.gzxc.cn:8081/products/resolve/all/ rfid0752615.htm

[16] VDC, Venture Development Corporation,

[17] Volkswagen (2009) http://www.reuters.com/article/pressRelease/ idUS135149+24-Mar-2009+MW20090324)

[18] Williams, D. "The Strategic Implications of Wal-Mart's RFID Mandate." Directions Magazine, July 28, 2004. Available online at www.directionsmag.com/article.php?article_id=629\&trv=1\&PHPSESSI $\mathrm{D}=\mathrm{a} 942 \mathrm{fc} 54 \mathrm{a} 33502601 \mathrm{eb} 2 \mathrm{cbbec} 3 \mathrm{fced} 74$. (Accessed on 20 March 2010). 